\title{
On Language Specification and Politeness Online in the Light of Microblog Headlines by People's Daily
}

\author{
Wang Ying, Xiao Jiugen* \\ Research Center of Language and Language Life, Jiangxi Normal University, Nanchang, China \\ Email address: \\ 1539847990@qq.com (Wang Ying),jxsdxjg666666@sina.com (Xiao Jiugen) \\ ${ }^{*}$ Corresponding author
}

To cite this article:

Wang Ying, Xiao Jiugen. On Language Specification and Politeness Online in the Light of Microblog Headlines by People's Daily. Science Innovation. Vol. 8, No. 1, 2020, pp. 18-21. doi: 10.11648/j.si.20200801.16

Received: January 13, 2020; Accepted: February 28, 2020; Published: March 17, 2020

\begin{abstract}
The official microblog account of People's Daily was first logged in on July 22, 2012. Same as the publications of People's Daily, it equally owns an unquestionable authority and mass character. Due to the distinctiveness and authority, the account was paid great attention to since its registration. Today, as the only official account of People's Daily, it enjoys an incomparable attention: according to the statistics, in 2019, the number of the followers of the account has reached 101 million, and the interaction in related topics enabled the government to communicate effectively with the masses, all of which contributed to the first place among all the official accounts concerning the influence online. Such a significant achievement is credited mainly to two aspects. One is the authority, for the account actively leads people to express politely, so that a more civilized online environment can be constructed. Another is responsibility, for it selects the internet phrases, so that the positive attitude can be spread, and our new era can be injected with vitality. The survey also shows that the Weibo (Chinese microblog) of People's Daily is not only rich in content and normative in language, but also pays special attention to the art of language use, which has attracted great attention from scholars, with nearly 10,000 or more research papers per year. Of course, with the growing development of Weibo today, the country must not only be a leader in the Weibo of People's Daily, but also other Weibo bloggers as platforms that can reflect segments of society. As long as management and regulation are strengthened, Weibo will definitely play an increasingly important role in the socialist two civilizations construction.
\end{abstract}

Keywords: People's Daily, Official Account, Headline Diction, Language Specification, Language Politeness

\section{从《人民日报》微博标题看网络语言规范和语言文明}

\section{王莹, 肖九根 ${ }^{*}$}

江西师范大学语言与语言生活中心, 南昌, 中国

邮箱

1539847990@qq. com（王莹），jxsdxjg666666@sina.com（肖九根）

摘要：《人民日报》官方微博自 2012 年 7 月 22 日开通以来，相较于其它纸质媒体而言，其权威性如同《人民日报》一样 不容置疑, 具有广泛的人民性。作为唯一的、且具关注度最高的官方微博, 由于其特殊性、权威性, 在起步时就受到 了大众的高度关注，拥有庞大的粉丝群。据统计，截至2019年止，《人民日报》微博关注的粉丝已达 1.01 亿，其话题 互动参与活动真正实现了与人民大众的有效沟通, 影响力在新浪全媒体排名榜中稳居第一。之所以有如此高的关注度、 影响力, 主要在于: 一是以其权威性对微博语言加以引导, 使之不断规范, 走向文明; 二是以其责任心对微博语言加 以选词, 使之在传递正能量中显示新时代蓬勃的生命活力。调查也显示, 《人民日报》微博不仅内容丰富, 语言规范, 还特别讲究语言的运用艺术, 这引起了学者们的极大关注, 每年的研究论文达近万甚至万篇以上。当然, 在微博日益 
发展的今天, 国家不仅要如《人民日报》微博这样的领军旗手, 还要反映社会生活的其它微博平台。只要加强管理与 规范，微博在进行社会主义两个文明建设中就一定能发挥越来越重要的作用。

关键词：人民日报，官方微博，标题选词，语言规范，语言文明

\section{1. 引言}

据调查统计, 截至2019年11月底, 微博月活跃用户达 到 4.97 亿, 日活跃用户增长数为 2.16 亿, 且 $94 \%$ 为移动用 户，较上年同期净增约 2100 万，每日阅读量 100 万十。1 随着微博平台功能的不断完善, 微博用户群开始稳定并保 持持续增长。作为活跃的网络社交平台, 其主要传播方式 是用户群体阅读、转发、评论等。在指尖阅读时代, 标题 对信息内容概况起到简明扼要的提示作用, 因此标题词语 的选择显得尤为重要。标题词语的选择, 不仅要在符合语 言规范的基础上求新, 切合时代发展的要求, 还要采用大 众喜闻乐见的形式, 能在文明用语中透视语言所蕴含的丰 富内容。

《人民日报》微博的产生, 也引起了学者们的特别关 注, 几年来出现了大量的研究文章, 甚至还有不少硕士或 博士学位论文。从期刊论文的数量看, 2016年9206篇, 2015、 2014、2013年均在万篇以上，就是2012年人民日报微博创 立这年,其数量也达 8 千有余; 论文内容主要集中于：一是 人民日报对新闻事件评论或报道的特点(包含对重大事件 的报道举例); 二是人民日报对于與论的引导; 三是探究 人民日报微博成功的原因; 四是人民日报对国家机关、企 业个人、特定事件等客体形象的构建。[1]作为具有权威性、 导向性的《人民日报》微博, 特别重视语言的规范性和标 题语言的艺术性, 虽偶有失误的情况, 但能及时地加以纠 正, 沿着健康的正确方向发展。

对于语言文字运用问题, 国家历来十分重视, 颁布了 很多有关法令文件, 提出了语言文字规范化的标准。早在 上世纪50年代, 《人民日报》发表了《正确使用祖国的语 言, 为语言的纯洁和健康而斗争》社论; 1997年, 国家语 委出版了《国家语言文字规范和标准选编》；2012年, 教 育部、国家语委颁布了《国家中长期语言文字事业改革和 发展规划纲要（2012-2020年）》; 2018年, 教育部、国 家语委印发了《国家语言文字工作委员会语言文字规范标 准管理办法 (2018年修订) 》, 等等。这些法令文件足以 说明, 无论社会、经济、文化以何种方式、何种程度变化, 国家对语言文字标准化、规范化的重视始终不变。加强国 家语言文字使用的管理规范工作, 任何时候都是十分必要 的, 在微博日益发展的今天更是如此。因此, 当下进一步 强化其管理与规范, 对于传递社会主义核心价值观、进行 两个文明建设都有重要意义。

\section{2. 《人民日报》微博标题与时俱进，选词在规 范中走向灵活}

《人民日报》官博无论语言表达、话语风格还是内容 结构, 都符合当代社会发展的需要, 打破了官媒一本正经
的刻板印象, 不再单靠话语的简洁概括取胜, 而是以灵活 多样的语言表达、别出心裁的话语风格、新颖独到的思想 内容于众多媒体中脱颖而出, 斩获大众的好评和关注。实 际上也是这样, “网络语言的大量出现丰富了既有的汉语 语言词汇体系。更收入了权威词典, 登入主流媒体, 甚至 对其他语言都产生了影响”[2],如《现代新华字典》（第七 版）就出现了“北漂”“给力”“团购”等新词语。网络语言有 着其它媒体难以取代的优势, 它能“丰富现有汉语表达形 式, 提升民众表达能力, 加快网络表达速度, 扩大覆盖范 围”。[3](p60-62)“网络语言爆发式增长是互联网快速普及 的结果, 也是网民真实情感诉求的线上展示”。[3](p60-62) 《人民日报》是党报, 书写国家意志, 代表中国形象。

因此, 《人们日报》报刊内容多以国家大事为主, 语言客 观严谨, 表达严肃谨慎。而其官方微博相对来说, 与大众 娱乐化、生活化联系紧密, 也轻松活泼, 不少流行词、口 语词、网络热词都可在标题中得到广泛运用, 其用语通俗 易懂, 更加贴近大众。例如, 12 月 23 日《人民日报》微博 发文: “井唐朝月半妹妹的发型\#【当国宝被重新配乐后, 画风突变】阿斯塔那墓葬群出土俑及文书、洛神赋图、唐 仕女俑......穿越千年, 沉静的国宝在摩登的音乐配合下, 向现代人娓娓道出自己的故事。翟看国宝用最fashion的 方式跟你说话! ”不少网友评论: “这种新型介绍文物的方 式值得推广! ”“画风变得有点可爱”“变得萌萌哒”。大家知 道, “胖”本指人体内含脂肪多, 安泰舒适, 古代也指祭祀 用的半边牲肉, 语出《仪礼·少牢馈食礼》。而“月半”二字, 意味圆月切半, 与人胖相类。《人民日报》官方微博含蓄 幽默地将“胖”字拆开, 符合网民心理世界与现实文化对 “胖”的抵触情绪。

除去幽默因素, 再来看其是否符合语言规范化原则。 首先, 所谓语言不规范, “其实应该区分为语言本身和语 言表达内容两个层面的问题。”[4]语言本身不符合标准现 象, 如网络中使用的字、词、句等言语成分打破了已有的 语言文字系统规则, 没有遵守规则标准或约定俗成原则, 也不起特殊的修辞表达作用, 就可以谓之不规范。人们很 难把“胖”说成“月半”这样的用语现象归之为规范的语言, 但是如果这种说法已经约定俗成了, 那么也就属于规范的 了, 因为“约定俗成”本身就是规范化的一个原则和标准。 [5]对于这种“不规范”的语言现象, 不仅其它网络平台大量 存在, 就是《人民日报》微博中也屡见不鲜。正如罗常培 所说的: “语言是社会组织的产物, 是跟着社会发展进程 而演变的”, “语言不是孤立的, 而是和多方面联系的”。[6] (p108-109)

有些语言用法虽然不符合语言学中的规范标准, 但是 只要进入言语交际领域, 为社会大众所接受和使用, 就值 得肯定。例如, “\#我的中国心\#手势舞挑战, 一起来打卡, 澳门回归祖国 20 周年, 为了祝福澳门, 祝福祖国! ”这个 
话题讨论次数已达 90.2 万, 阅读次数 6.9 亿, 且在不断增长。 又如《人民日报》微博所说的: “就差你了”“\#微盘点\#”话 题, 阅读次数 88.6 亿, 讨论次数 797.9 万, 原创人数 12.8 万; “\#你好, 明天\#”话题, 阅读次数 117 亿, 讨论次数 735.7 万, 原创人数 3.9 万; “\#人民锐评\#”话题, 阅读次数 20.4 亿, 讨 论次数 35.3 万; “\#人民微评\#”等常用话题阅读、讨论次数 往往能引起人们极大的关注。《人民日报》标题话题建构, 不仅反映影响着人们的思维和认知, 而且其语言传播具有 很强的代表性、时效性和导向性。因此, 《人民日报》微 博用语必须关注语言传播的规范性。新闻网络用语特别是 媒体微博用语生存在瞬息万变的互联网传播环境中, 语言 表达追求个性出奇, 其新颖的表达方式往往能吸引大众眼 球。反之, “循规蹈矩”的语言表达方式，在新时代语言文 化背景下有可能处于一个“待机”静默状态。《人民日报》 微博标题选词, 一方面在重塑语言规范意识, 另一方面又 在引领语言时代风尚。其在注重信息内容本身的时效性、 准确性的同时, 关注着语言的规范与文明, 如传播的能量 越大, 其接受者长此以往在话语参与下也就自觉而然地处 于语言规范文明之中了。

2019年12月18日，《人民日报》微博中写道: 【画风 变了! \#东风快递硬核宣传片要火\#[威武] ““扎根、过硬、 奋斗、奉献'是高原火箭兵传承 50 年的精神。是这样的精 神, 赋予每一位火箭兵忠诚; 是这样的精神, 使他们在雪 域高原、戈壁大漠中肩负起保家卫国的责任。高原火箭兵 震撼出境, 诠释中国硬核力量。”其中“扎根、过硬、奋斗、 奉献”精准地表达了“兵哥哥”的特质和“为人民服务”的精 神, 赞扬他们时便用了“硬核”这个网络流行语 (2019年)。 “硬核”原指一种力量感强、节奏激烈的说唱音乐风格, 随 后其义进一步引申，人们常用“硬核”形容“很厉害”“很彪 悍”“很刚硬”的精神作风。《人民日报》微博曾多次以“硬 核”为标题, 如: 【硬核视频! \#驻港部队海上军事训练井[威 武]】、100岁之前都是童年！\#2019年硬核大爷大妈\#】、

【硬核! \#语文老师上核舟记直接画核舟\#】等。可见, “媒 体的语言影响力是巨大的, 媒体对社会语言生活发挥着主 导作用, 带领着语言向前发展。”[7](p34-38)作为以语言文 字为主要传播手段的《人民日报》微博平台, 也是语言文 字规范的一个重要领域。

毫无讳言, 在信息内容密集输出时, 语言不规范的现 象会时常存在于网络平台中。作为官方微博, 《人民日报》 也不可避免地出现一些纰漏, 造成不规范的语言现象。如 12 月 21 日， 《人民日报》微博上发表的《\#90后出纳员 819 次挪用公款\#, 称看到钱就像失心疯》一文, 编者将“Ukey” 错成了“Ukeg”。这种意思截然不同的用词一经发出, 就引 起了相关人员的批评。随后，人民日报微博编者立即改正 了错误。可以说, 无论任何内容要“实现传播, 必然要求语 言规范。语言越规范, 传播越广泛”。[8](p43-45)越是规范 的语言内容, 其受众面越广, 大众的信任度越高, 就越能 在网络中生根、开花、结果。

又如, 2019年8月24日, 《人民微博》标题: 【\#清华 女生手绘军训手帐引发回忆潮\#: 当年流下的汗都是现在 笑出的泪[悲伤]】中“手帐”使用“帐”，而2018年1月24日微 博:【99个手绘简笔画小盆栽, 哄娃手账必备, Mark!】 中则是“手账”，其中的“账”与“帐”用法模糊，使人无所适
从, 难以分辨。其实, “用帐而不用账, 已规范几十年 了。”[9](p89-90)但是, 2004年第10版 (双色本) 《新华字 典》、2002年修订的第3版（增补本） 《现代汉语词典》 又收有帐、账。如此摇摆不定, 反反复复, 无形中给人们 学习和使用带来了麻烦。这就要求《人民日报》微博要在 正确而科学地使用语言文字方面树立旗帜, 对语言文字抱 着一种“锬铢必较”的精神, 确定统一的规范标准, 使其保 持相对的稳定, 这样有利于人们的学习与运用。

\section{3. 《人民日报》微博标题破立相彰, 选词在文 明中熠熠生辉}

从微博中看语言文明, 以小见大, 管中窥豹, 这有助 于人们更好地了解社会。一谈起网络语言文明, 有人就紧 皱眉头, 认为这种非现实交际不需遵从礼貌原则, 因而语 言不规范也表现在语言表达上, 如言语粗俗、鄙陃、不文 雅等。历年来, 不少网络热词一开始便显示其自身的生命 力, 而那些低俗不堪的词语则在海量信息中自然地湮没, 遭到淘汰。因此, 人们“对失范因由的深入探究有助于把 好网络脉搏, 从而更好地对其进行规范”。[10]

《人民日报》微博关注社会生活, 涉及社会生活的方 方面面，展示着不同阶层、不同职业、不同年龄、不同性 别的丰富内容。如 12 月 23 日的微博: 【\#孙小果再审案宣 判\#: 孙小果被判死刑, 剥夺政治权利终身, 并处没收个 人全部财产】, 面对这样一个穷凶极恶、罪不可恕的罪犯, 网友评论最多的是“死刑太便宜他了”“死刑是他的归 宿”“正义也许会迟到, 但永远不会缺席”等, 我们并未看 到以往的漫天咒骂或极端控诉。由此可见, 网民以更理性、 更文明的态度面对社会事件。又如12月13日的微博：【\# 黑龙江警方查获野生熊掌214只\# 它们本不该是这样[伤 心]，网友一致以赞扬的语言评论“没有买卖就没有伤 害”“抵制野生动物制品, 关爱野生动物, 人人有责! ”当 然, 语言文明的构建不是一朝一夕的事, 而是体现在长期 的生活事件细节之中, 如上述评论。一个个温暖纯良的个 体在网络中放大、扩散, 如同空气净化器一般, 让每个人 在一呼一吸中为文明语言所熏陶, 所浸染。《人民日报》 微博的语言表达, 既文明又贴近生活, 网友平等交流易拉 近彼此间的心理距离, 并在此基础上通过规范文明的语言 文字传播核心价值观，树立正面导向。从语言文明到文明 语言, 这是一个循环往复的螺旋式上升过程。2014年3月, 习近平主席在柏林会见德国汉学家、孔子学院师生代表时 指出: “沟通交流的重要工具就是语言。一个国家文化魅 力, 一个民族凝聚力主要通过语言表达和传递。掌握一种 语言就掌握通往一国文化的钥匙。”可以说, 《人民日报》 微博标题选词巧妙地运用了语言这把钥匙开启全民智慧 的文明大门。语言的规范使用有助于提升人们思维的清晰 度和严谨度, 提高人们的认知水平和审美情趣。在这样的 语言环境下, 语言文明自然起到先导作用。为了进一步提 高关注度, 微博就要“在传播内容上,追求悬念叙事、彰显 多元主体、培养读者写作,要为读者量身打造阅读主题,并 照顾读者的叙事偏好, 用他们喜爱的讲故事方式娓娓道 来。”[11]毫无疑问，《人民日报》微博标题选词在彰显大 
众文化, 将语言文明提升到一个新的高度方面起到了率先 垂范的作用。众所周知, “中华民族数千年的文化保存至 今, 其中规范的科学的语言文字, 是维系这种文化的纽带”, [12]（p2）因为语言的文明不仅仅是一种文化的文明, 更 是一个民族社会成员素养的反映。

《春秋谷梁传》云: “人之所以为人者, 言也。”惟有语 言, 人才与其它动物分道扬镳, 其它动物是没有语言的。语 言能传递信息，是人类进行思维活动的载体。而传递信息、 进行文明交际是离不开语言规范的, 所谓没有规矩难以成方 圆就是这个道理。有的学者认为, “规范化是社会语言共同 体的需要。信息社会更需要规范化,因为信息化社会就是要求 信息交际的高速度、高效率。没有规范化,高速度和高效率也 就无从谈起”。[13](p3-9)语言规范不仅仅关乎个体，还关乎 整个国家政治、经济、文化的发展，这是因为语言文明是一 个国家文明的表征。可以这样说, 守住的是语言文明, 赢得 的是国家未来。《人民日报》微博标题选词作为社会发展的 一面镜子, 其用语折射出不同时代的文化特色和科技进步, 如“买买买后”“骄傲转发”“胖五”（长征五号）等, 这在一定 程度上既规范了网络用语, 也维护了网民的权益。当然, 尽 管“网络语言是网民话语权的重要阵地, 但在这个阵地上, 必 须传播导向正确和积极和谐的文化”, [14]否则话语权随时有 被剥夺的可能。不过, 语言规范是一项长期而艰巨的任务, 因其“规范性是相对的，而语言的变化和发展是绝对的”，并 且“社会的发展总的趋势是越来越走向语言和文化的多元化 和平民化, 而不是走向单元化和精英化”。[15](p73-78)

\section{4. 结语}

随着网络微博的快速发展, 网民的用语也良莠不齐, 对之规范不仅很有必要, 而且也很迫切。实际上, “语言 本身就是一种制度, 一种规范, 语言使用不能不尊重语言 制度, 遵守语言规范。”[16]只有这样, 才能保证其沿着正 确的道路健康发展。在网络平台上，《人民日报》微博所 体现出来的语言规范和语言文明已经树立了一个很好的 样板, 发挥了正确的示范引导作用。如果每个媒体人都能 像《人民日报》那样富有责任心和担当力, 闻过则喜, 闻 过则改, 并时刻警示自己, 树立语言规范意识, 这无疑会 提高语言的文明程度, 发挥语言的交际作用。

语言是服务社会, 满足人们交际需要的。语言从社会 中来, 又回到社会中去, 因而具有强大的生命力。而网络 语言有其自身独特的鬼米力, 对之应该谨慎处理。对待网络 语言, 既要抱着一种宽容的态度, 又要本着一种负责的精 神。网络中那些不文雅、低俗用语, 必须坚决摒弃掉, 不 能任其自由泛滥。要让大众接受传递正能量的规范语言、 文明语言的熏陶, 在网络中自觉维护网络文明。人们还应 辩证看待、客观分析新的语言现象, 要按照国家出台的相 关法令文件精神, 做好语言规范工作。与此同时, 还要进 一步强化权威媒体如《人民日报》微博语言的样板示范效 应。可以预见, 只要国家对微博加强管理与规范, 其在社 会主义物质文明与精神文明建设中就一定能够发挥越来 越重要的作用。

\section{注释}

1. 漆辛夷,一财网. 微博第三季度总营收33.18亿, 日活跃 用户增长至2.16亿.

[EB/OL].https://baijiahao.baidu.com/s?id=1650186807 302635327\&wfr=spider\&for=pc，2019-11-14.

2. 刘志勇. 习近平同德国汉学家、孔子学院师生代表座 谈.[EB/OL].news.cntv.cn/2014/03/29/ARTI139610783 6084471.shtml,2014-3-9.

\section{参考文献}

[1] 陈文博.我国人民代表大会网络形象初探一一基于人民日 报微博的内容分析 [J]. 人大研究,2019(05).

[2] 李玮,禇建慧.新兴媒体对语言文化的影响 [J].新闻与写 作,2018(03).

[3] Zhao Yue. On the influence of the development of network language on Chinese in the new era $[\mathrm{J}]$. Henan agriculture, 2019 (30).

[4] 张 黎.大家谈·语言文字规范那些事儿: 网络语言, 到底该 规范什么[N].光明日报, 2019-7-8.

[5] 叶蜚声、徐通铭. 语言学纲要 [M]. 北京: 北京大学出版 社.1997.

[6] 罗常培.语言与文化[M].北京: 北京出版社, 2003.

[7] 李宇明.大众媒体与语言 [A].姚喜双,郭龙生.媒体与语言: 来 自专家与明星的声音[C].北京:经济科学出版社,2002.

[8] 姚喜双.加强媒体语言研究——需要解决的几个问题 [J].语 言文字应用,2005(03).

[9] 沈奇喜.咬文嚼字[J].学语文,2019(04).

[10] 陈春雷.从失范走向规范——关于网络语言影响及规范策 略的思考[J].学术界,2011(04).

[11] 许林,邱新有.移动互联网语境下报告文学的平台创新与内 容策略[J].江西师范大学学报(哲社科版),2018(03).

[12] 于根元.网络语言概说[M]. 北京: 中国经济出版社, 2001 .

[13] 陈原.变异和规范化[J].语文建设,1987(04).

[14] 贾小霞.网络语言规范与和谐社会文化的构建 $[\mathrm{J}]$.山西财经 大学学报,2014,36(S1).

[15] 沈家煊. 词典编纂 “规范观” 的更新 [J]. 语言教学与研 究,2005(03).

[16] 李宇明.语言技术对语言生活及社会发展的影响[J].中国社 会科学,2017(02). 